\title{
Problèmes de prévision d'écoulements diphasiques dans l'exploitation des hydrocarbures souterrains
}

\author{
Two-phase flow prediction problems \\ in underground hydrocarbon recovery projects
}

\author{
Jean-Michel Fitremann
}

École Nationale Supérieure de Mécanique, Nantes

\section{Introduction}

Les combustibles se présentent sous forme de mélanges d'hydrocarbures très complexes maintenus dans une roche poreuse à pression élevée. Dans l'extraction naturelle, la détente produit l'apparition de gaz libre soit dans la roche-réservoir, soit dans le puits. Les gisements comportent parfois aussi du gaz naturel, soit en partie, soit en totalité. Fréquemment, une fraction du gaz peut se condenser par détente (fluide rétrograde) et une extraction de gaz peut présenter des proportions variées de condensat. Le tableau 1 donne quelques exemples et les propriétés physiques des effluents.

En ce qui concerne les puits d'huile, le problème est essentiellement celui de la prévision de la relation débit-différence de pression afin de mieux prévoir les investissements de surface compte tenu des durées de vie prévues des gisements. On peut aussi injecter du gaz à la base du puit pour augmenter son débit (gas-lift) dans le régime où le gradient de pression diminue avec le débit de gaz; dans ce cas, une bonne prévision du phénomène permet de mieux évaluer l'incidence économique du procédé.

\begin{tabular}{|c|c|c|c|c|c|}
\hline \multicolumn{4}{|l|}{ Tableau 1} & \multicolumn{2}{|c|}{ Exemple de propriétés physiques } \\
\hline & & \multicolumn{2}{|c|}{ Puits AGM 4} & \multicolumn{2}{|c|}{ Gas line FRIGG St FERGUS } \\
\hline \multicolumn{2}{|c|}{$\begin{array}{l}\text { Pression de fond } \\
\text { Pression en tête de puits } \\
\text { ou en début de ligne } \\
\text { Température } \\
\text { Pression en fin de ligne } \\
\text { Température }\end{array}$} & 79 bars & $\begin{array}{c}24.5 \text { bars } \\
52^{\circ} \mathrm{C}\end{array}$ & $\begin{array}{c}\text { (140 bars) } \\
100 \text { bars } \\
17^{\circ} \mathrm{C}\end{array}$ & $\begin{array}{l}60 \text { bars } \\
7^{\circ} \mathrm{C}\end{array}$ \\
\hline $\begin{array}{l}\text { Masses } \\
\text { volumiques }\end{array}$ & $\rho_{L}$ & $\begin{array}{r}722 \mathrm{~kg} / \mathrm{m}^{3} \\
98 \mathrm{~kg} / \mathrm{m}^{3}\end{array}$ & $\begin{array}{r}766 \mathrm{~kg} / \mathrm{m}^{3} \\
24 \mathrm{~kg} / \mathrm{m}^{3}\end{array}$ & $\begin{array}{r}725 \mathrm{~kg} / \mathrm{m}^{3} \\
92 \mathrm{~kg} / \mathrm{m}^{3}\end{array}$ & $\begin{array}{r}725 \mathrm{~kg} / \mathrm{m}^{3} \\
55 \mathrm{~kg} / \mathrm{m}^{3}\end{array}$ \\
\hline $\begin{array}{l}\text { Vitesses } \\
\text { débitantes }\end{array}$ & $\begin{array}{l}U_{L} \\
U_{G}\end{array}$ & $\begin{array}{l}0.8 \mathrm{~m} / \mathrm{s} \\
1.7 \mathrm{~m} / \mathrm{s}\end{array}$ & $\begin{array}{l}0.73 \mathrm{~m} / \mathrm{s} \\
6.8 \mathrm{~m} / \mathrm{s}\end{array}$ & $\begin{array}{c}2.410^{-3} \mathrm{~m} / \mathrm{s} \\
3.8 \mathrm{~m} / \mathrm{s}\end{array}$ & $\begin{array}{c}2.8 .10^{-3} \mathrm{~m} / \mathrm{s} \\
7 \mathrm{~m} / \mathrm{s}\end{array}$ \\
\hline Viscosités & $\begin{array}{l}\mu_{L} \\
\mu_{G}\end{array}$ & $\begin{array}{l}0.9610^{-3} \mathrm{PQ} \\
14.710^{-6} \mathrm{PQ}\end{array}$ & $\begin{array}{l}1.3410^{-3} \mathrm{Pl} \\
13.210^{-6} \mathrm{Pl}\end{array}$ & $\begin{array}{l}0.6610^{-3} \mathrm{Pl} \\
14.510^{-6} \mathrm{Pl}\end{array}$ & $\begin{array}{l}0.7610^{-3} \mathrm{PQ} \\
12.610^{-6} \mathrm{Pl}\end{array}$ \\
\hline \multicolumn{2}{|c|}{ Tension interfaciale $\sigma$} & $0.012 \mathrm{~N} / \mathrm{m}$ & $0.022 \mathrm{~N} / \mathrm{m}$ & $0.0095 \mathrm{~N} / \mathrm{m}$ & $0.0155 \mathrm{~N} / \mathrm{m}$ \\
\hline \multicolumn{2}{|c|}{$\begin{array}{l}\text { Diamètre intérieur } D \\
\text { Longueur de l'écoulement } L \\
\text { Pentes } \\
\text { Configurations } \\
\text { Gradient thermique }\end{array}$} & \multicolumn{2}{|c|}{$\begin{array}{c}0.062 \mathrm{~m} \\
2490 \mathrm{~m} \\
90^{\circ}\end{array}$} & \multicolumn{2}{|c|}{$\begin{array}{c}0.775 \mathrm{~m} \\
350 \mathrm{~km} \\
\pm 2^{\circ}+90^{\circ}-90^{\circ} \\
\text { ratifié }+ \text { Gouttelettes }\end{array}$} \\
\hline
\end{tabular}


Pour le transport diphasique, les problèmes sont nombreux : prévision des gradients de pression et des contenus de liquide de lignes en terrain accidenté fonctionnant en régime instationnaire, conditions d'apparition de bouchons, volume et fréquence de ceux-ci, mouvement des râcleurs, couplage des lignes "horizontales" et verticales... Les problèmes peuvent être de nature très différentes selon qu'il s'agit d'huile + gaz où la configuration prédominante est la configuration à bouchons et les lignes souvent assez courtes (réseaux de collectes) ou des gaz plus ou moins chargés en condensat où la configuration est le plus souvent stratifiée avec des gouttelettes.

Bien entendu, la prévision des écoulements nécessite une bonne connaissance des propriétés physiques et thermodynamiques des effluents dans les conditions de l'écoulement. La transposition des mesures faites à l'équilibre ou en détente rapide au laboratoire est assez incertaine. Comme nous le verrons plus loin, les propriétés interfaciales jouent un rôle très important et les renseignements sur celles-ci sont parfois incomplets.

\section{Ecoulements diphasiques verticaux}

\section{Configurations d'écoulement}

Les configurations d'écoulement le plus souvent rencontrées sont la configuration à bulles et la configuration à bouchons aérés (fig. 1). La deuxième est la plus fréquente dans le cas d'huiles à forte teneur en gaz dissous en général encontrée vers la fin de la vie d'un gisement et dans le cas du gas-lift. Il est rare que l'on atteigne l'écoulement annulaire à gouttelettes, mais ce cas peut se produire pour des teneurs très élevées en gaz (supérieures à $500 \mathrm{~m}^{3}$ standard $/ \mathrm{m}^{3}$ d'huile environ et des faibles pressions de tête de puits) ; il se produit également ce type d'écoulement dans les puits de gaz à condensat et les parties verticales (riser) des conduites de transport.

Dans le cas des puits déviés par rapport à la verticale, l'aspect de l'écoulement n'est pas notoirement différent, bien que les lois de gradient de pression soient modifiées. Les transitions de configuration sont déplacées, mais il y a peu de résultats sur ce sujet à l'heure actuelle.

$\mathrm{La}$ configuration à bouchons aérés se produit pour un nombre de Bond

$$
\frac{\Delta \rho g D^{2}}{\sigma} \geq 140
$$

dans le cas inverse, la configuration est à bouchons pratiquement sans bulles [1] [2]. Cette valeur limite correspond à des petites valeurs de $\nu_{L}\left(\sigma / \rho_{L}\right)^{-3 / 4} g^{1 / 4} \sim 10^{-3}$, valeurs usuelles dans les cas typiques d'extraction d'huiles. Dans le cas d'huiles de forte viscosité, la valeur limite de 140 pour le nombre de Bond doit être modifiée.

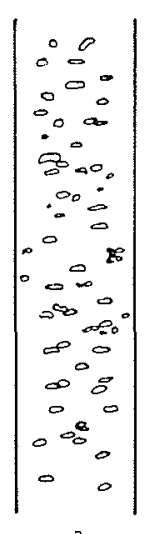

a

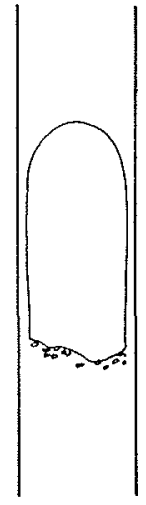

b

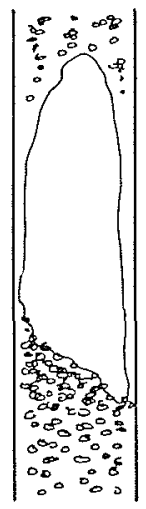

$c$

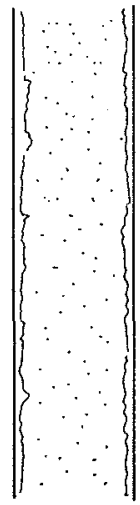

Figure 1. - Configurations d'écoulements verticaux.

a - à bulles

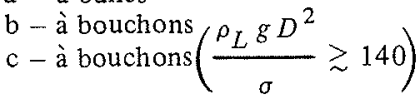

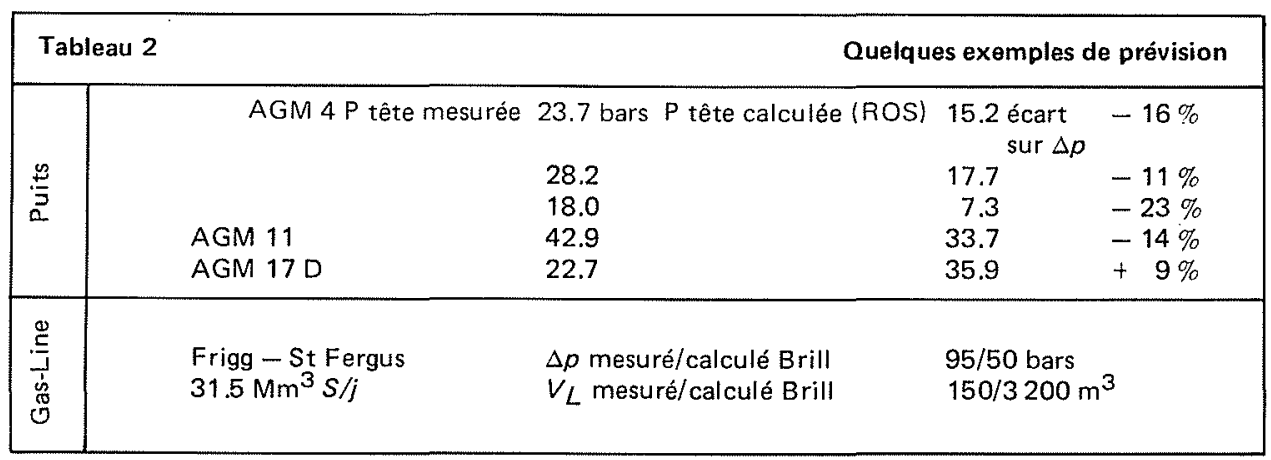




\section{Ecoulement à poches}

Bien que la prévision détaillée d'un écoulement à bulles laisse encore beaucoup à désirer, le modèle [2] donnant le gradient de pression dans cette configuration a une précision suffisante dans les cas usuels. Par contre, pour l'écoulement à bouchons, les résultats sont assez médiocres : en effet, les corrélations de ce modèle sont basées sur des mesures de glissement par traceurs qui peuvent être faussées lorsqu'il y a des écoulements de retour importants comme c'est le cas dans cette configuration (voir exemples du tableau 2).

La figure 2 montre le détail d'un tel écoulement : on trouve un film liquide descendant le long des poches de gaz $^{(1)}$ dont l'impact sur le bouchon de liquide entraîne du gaz au sein de celui-ci. Les bulles entrainées ont une vitesse ascensionnelle inférieure à celle des poches si la condition (1) est réalisée et sont captées par la poche suivante. Les dimensions des poches et des bouchons sont aléatoires mais la vitesse de déplacement géométrique de ceux-ci est quasi-déterministe avec une loi du type

$$
U=A\left(U_{G}+U_{L}\right)+B U_{\infty}
$$

où $U_{\infty}$ est la vitesse d'une poche dans une conduite où le liquide est au repos. Dans nos expériences $A=1.15$ et $B=1,67, U_{\infty}=0.35 \sqrt{g D}$ [1] [3] [4]. Les longueurs maximales observées des poches et des bouchons sont environ deux fois leur valeur la plus probable. Ces grandeurs varient rapidement avec la vitesse du gaz mais il est difficile de mettre en évidence une variation systématique avec la vitesse du liquide [1] [3] (fig. 4).

Pour des faibles valeurs de $U_{G}$, nous avons rencontré des difficultés dans la reproduction des conditions expérimentales : le taux de présence de bulles dans le bouchon dépend fortement des propriétés de coalescence du mélange et a une influence notable sur les gradients de pression mesurés dans les mèmes conditions de débit et de propriétés physiques usuelles. La tension interfaciale ne semble pas suffisante pour déterminer ces propriétés. Aux vitesses élevées, cette dispersion diminue (fig. 3).

La mesure du taux de présence des bulles et des poches séparément est possible par le traitement de signaux issus de sondes de présence locales [5] [6] [7]. Les résultats peuvent être inclus dans un modèle statistique de l'écoulement afin de prédire des grandeurs intéressantes: par exemple, pour des installations de séparation de phases calculées pour décanter les bouchons de liquide, il est intéressant de connaître le débit de liquide dans un bouchon. On peut montrer qu'il vaut

$$
\begin{aligned}
U_{B}=\left(1-<\alpha_{B}>\right) & \left(U_{G}+U_{L}\right) \\
& -<V_{\infty} \alpha_{B}\left(1-\alpha_{B}\right)>
\end{aligned}
$$

[8] [9]

pendant des intervalles de temps de durée moyenne

$$
\alpha_{0}=\alpha_{P}(r=0)
$$

(1) Relativement à celles-ci. Dans un repère absolu, la vitesse du film liquide peut-être dirigée vers le bas ou vers le haut selon la vitesse de déplacement des poches.

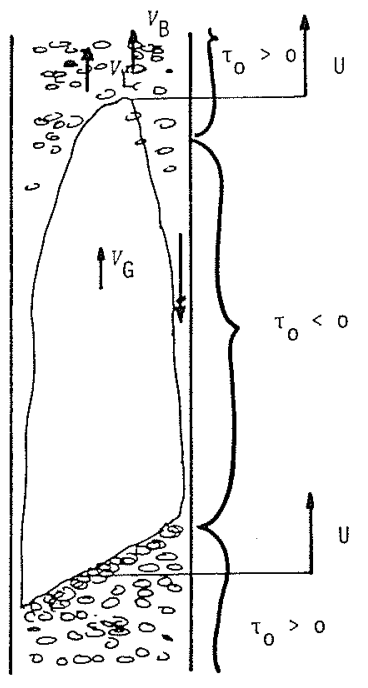

Figure 2. - Ecoulement à bouchons - Détail

$V_{B}>V_{L} \quad U>U_{G}$

Il est encore trop tôt pour avoir des modèles de prévision de $\alpha_{0},\left\langle\alpha_{B}\right\rangle, \bar{L}_{B}$ et $\bar{L}_{P}$ extrapolables à de nombreuses conditions de champs. Par ailleurs, la mesure directe du frottement pariétal instantané est nécessaire pour comprendre les mécanismes de perte d'énergie dans ces écollements. Des mesures, dont nous donnons un exemple (fig. 5) sont en cours au LEMTA (Nancy) et au LMEF (Orsay) pour étudier ces propriétés de l'écoulement [8].

\section{Ecoulement annulaire à gouttelettes}

La transition vers l'écoulement annulaire se produit par disparition de plus en plus fréquente des bouchons de liquide lorsque la vitesse du gaz croît.

En général, une fraction du liquide est entraînée sous forme de gouttelettes. La fraction entraînée est difficile à prévoir, mais les résultats [11] que nous avons utilisés semblent donner de bons résultats malgré le peu de paramètres mis en jeu; ils ne sont sans doute pas transposables à d'autres mélanges que ceux voisins de l'eau et de l'air. A l'aide de cette corrélation et d'une corrélation de frottement interfacial [12] on peut construire un modèle permettant d'évaluer le gradient de pression, le taux de présence de liquide et les propriétés des gouttelettes. Ce modèle donne des résultats comparables aux valeurs expérimentales obtenues en laboratoire [1].

\section{Transport diphasique}

\section{Transport du brut}

Les propriétés physiques du mélange sont celles indiquées pour les puits de pétrole, mais le diamètre des conduites peut varier selon le nombre de puits qui alimentent la collecte. De même, le profil longitudinal de la 


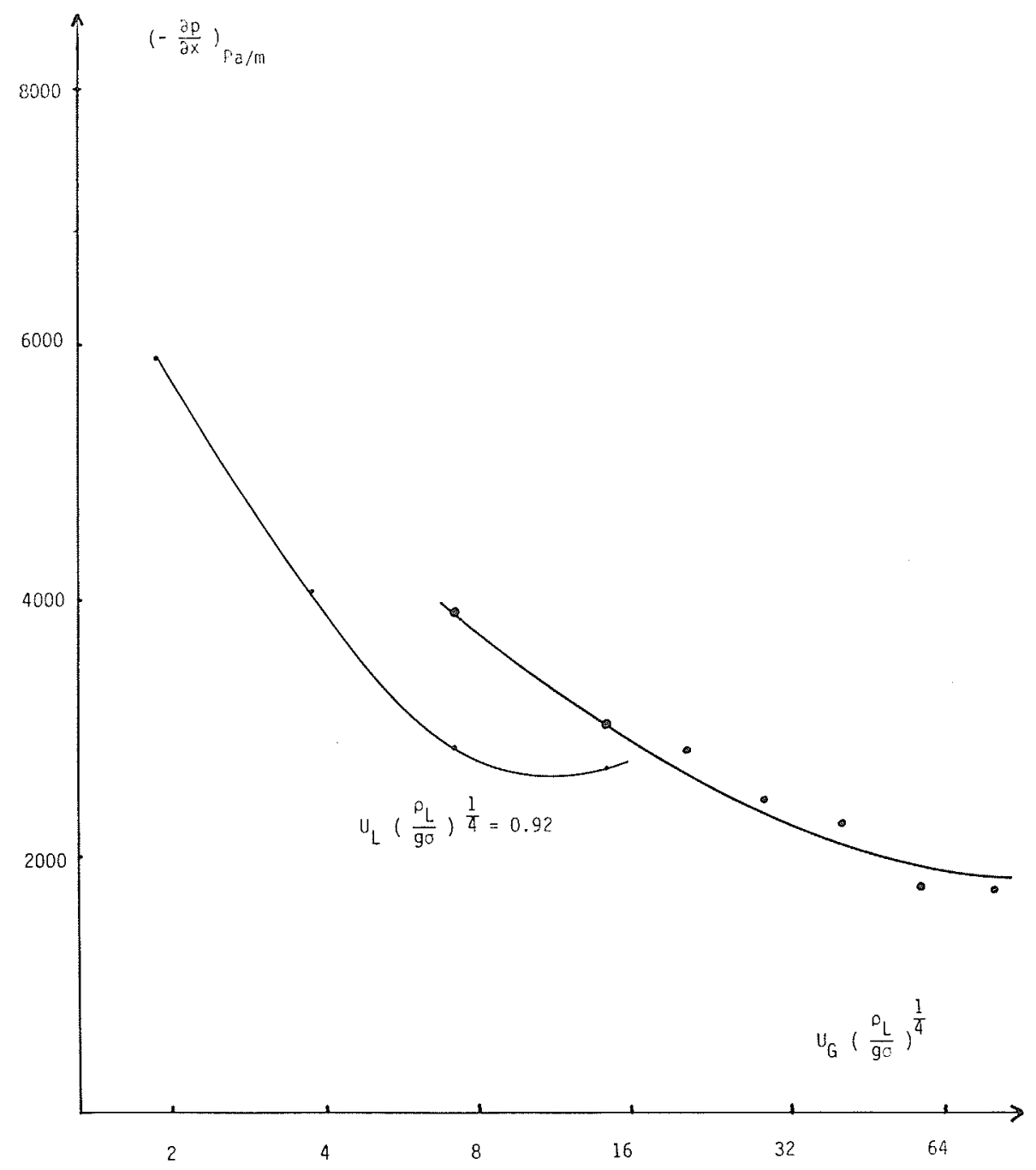

Figure 3. - Mesures de građient de pression dans des conditions identiques en écoulement à bouchons d'après [1] et [3]. Les différences résultent des différences sur le taux de présence des bulles $a_{B}$.

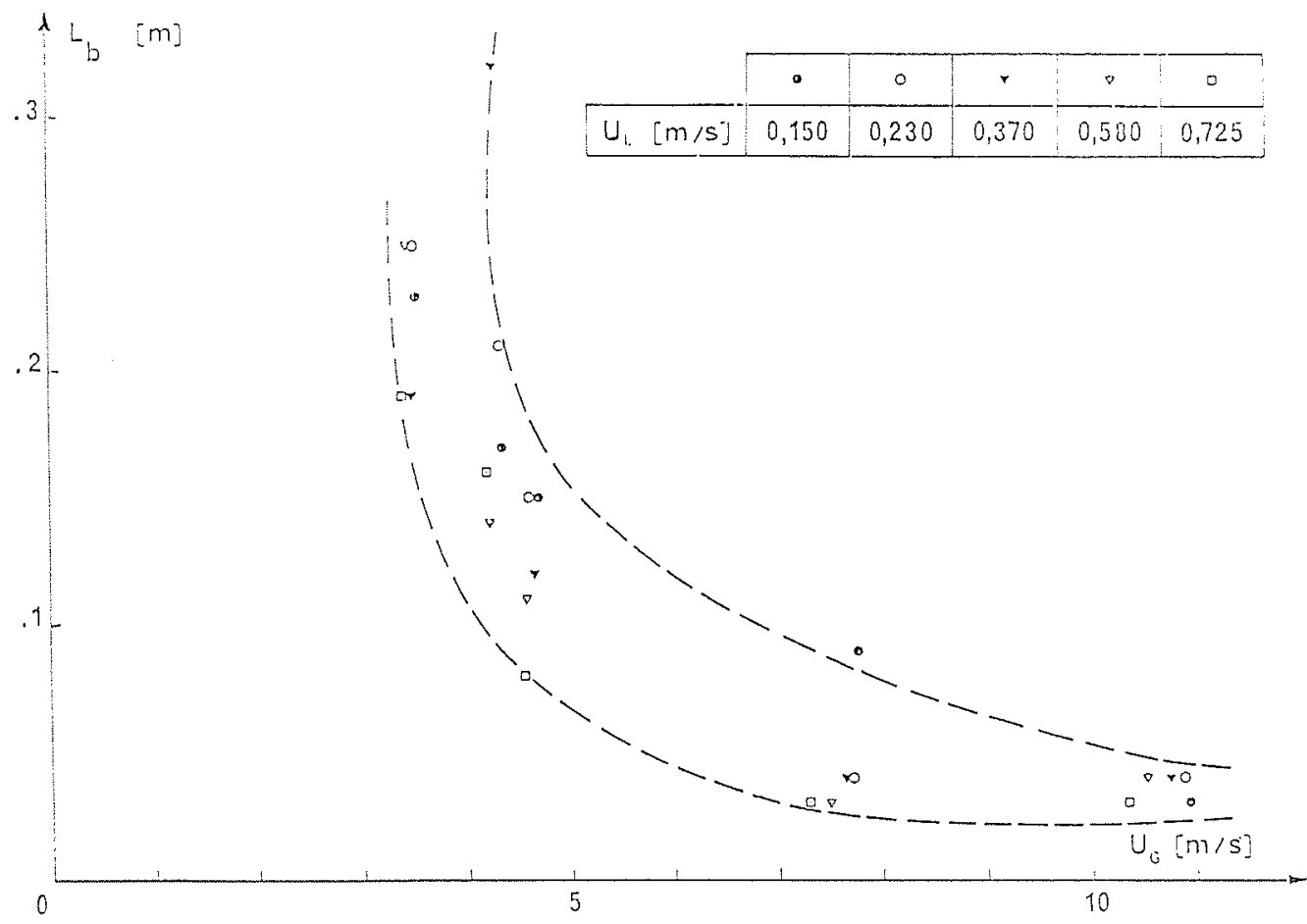

Figure 4. - Longueur moyenne des bouchons d'après [3]. 


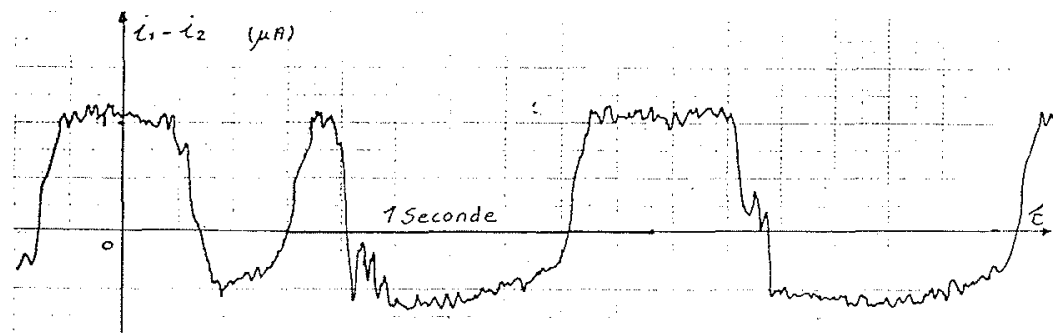

Figure 5. - Frottement pariétal en écoulements à bouchons d'après [8].
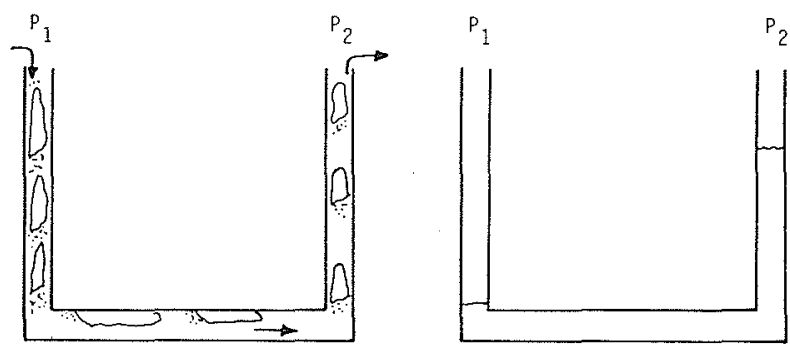

Figure 6. - Arrêt d'une ligne diphasique. La ligne peut ne redémarrer qu'avec une différence de pression très supérieure à $P_{1}-P_{2}$.

ligne peut être quelconque, suivant le terrain traversé : des pentes de $\pm 5^{\circ}$ ne sont pas rares et des tronçons verticaux peuvent s'intercaler à divers endroits de la ligne. La configuration prédominante est celle à bouchons, $y$ compris dans les parties descendantes; parfois l'écoulement y est stratifié si la teneur en gaz est suffisante. Les propriétés "diphasiques" de ces réseaux de collecte sont souvent déconcertantes: les différences de pression peuvent diminuer considérablement lorsque le débit augmente ou lors d'une restriction de section d'une portion de la ligne; il peut $\mathrm{y}$ avoir des oscillations de débit entre divers tronçons du réseau (dues aux "résistances négatives" toujours possibles en diphasique); il peut aussi y avoir blocage total d'une ligne fonctionnant parfaitement en régime dynamique (fig. 6). Comme nous consacrons une conférence à l'écoulement à bouchons horizontal, nous reportons le lecteur à celle-ci pour plus de détails [13] [14] [15].

\section{Transport de gaz}

L'exemple donné dans le Tableau 1 est un cas particulier de très faible teneur en condensat. Cette teneur est très variable selon les gisements. L'extraction offshore rend l'évacuation diphasique quasiment obligatoire.
La configuration est presque toujours stratifiée avec en général des gouttelettes. Il peut, dans certaines conditions de débit occasionnelles, se produire des bouchons dans les parties ascendantes à forte pente ou les tronçons verticaux (risers). Le problème est alors de protéger les installations de traitement ou de compression de l'arrivée éventuelle brutale de liquide (fig. 7). pour :

La prévision $d u$ régime permanent est importante

- le calcul du débit maximum de la ligne

- la prévision de l'arrivée régulière de liquide

- la prévision de la possibilité de bouchonnage des tronçons précédent des installations de traitement, compression, etc.

-- l'évaluation des débits maximum instantanés de liquide en cas de bouchons

- l'évaluation du contenu de liquide de la ligne pour la prévision des volumes obtenus lors d'un râclage

- idem pour la prévision du volume recueilli lors d'un changement de régime.

Par ailleurs, les phénomènes transitoires sont importants dans les lignes à gaz pour plusieurs raisons :

1 - le débit varie continuellement selon la demande, le régime permanent est rarement atteint. La pression peut varier également au gré de l'utilisateur de la ligne;

2 - les râclages annulent le contenu de liquide d'une ligne périodiquement ; celle-ci est donc fréquemment en cours de "remplissage" ; parfois, lorsque le volume de liquide en régime permanent paraît trop élevé (pertes de charge trop fortes, contenu de liquide supérieur aux organes de réception de celui-ci), le râclage a lieu avant d'atteindre le régime permanent: dans ce cas le régime est toujours transitoire (fig. 8) ;

3 - lors d'un râclage, le bouchon de liquide se déplace à vitesse variable, notamment lors du passage dans un tronçon vertical. Le bouchon de liquide a luimême un volume variable. Le volume de liquide recueilli correspond au film liquide ; la connaissance du taux d'entraînement des gouttelettes est importante (fig. 9).

On est alors amené à traiter plusieurs problèmes

G L

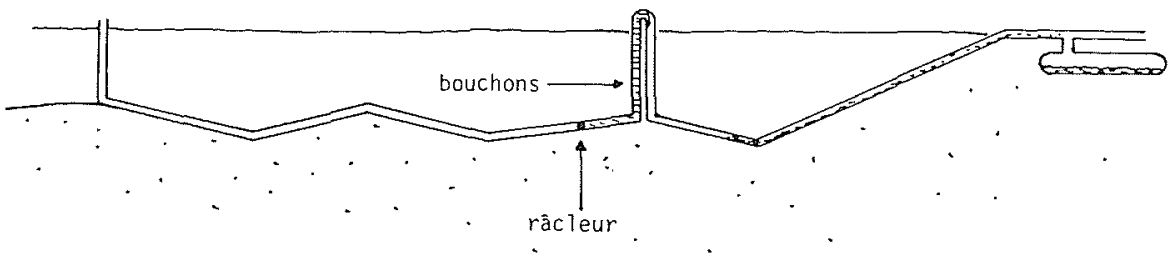

Figure 7. - Ligne de transport de gaz diphasique. 

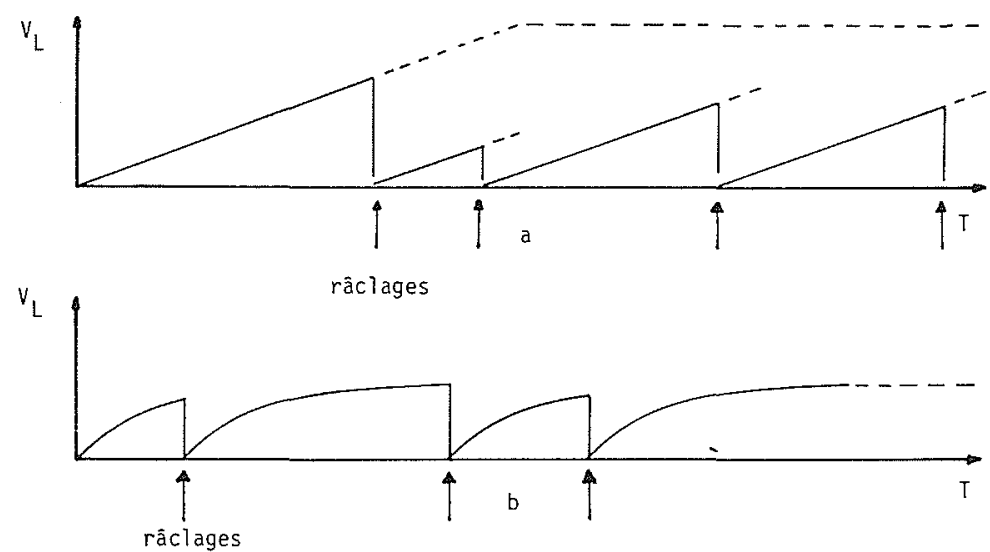

Figure 8. - Volume de liquide (sous forme de film) d'une ligne de transport imaginé à débit de gaz constant, avec raclages irréguliers : $a$ - Faible débit ; b - Débit élevé.

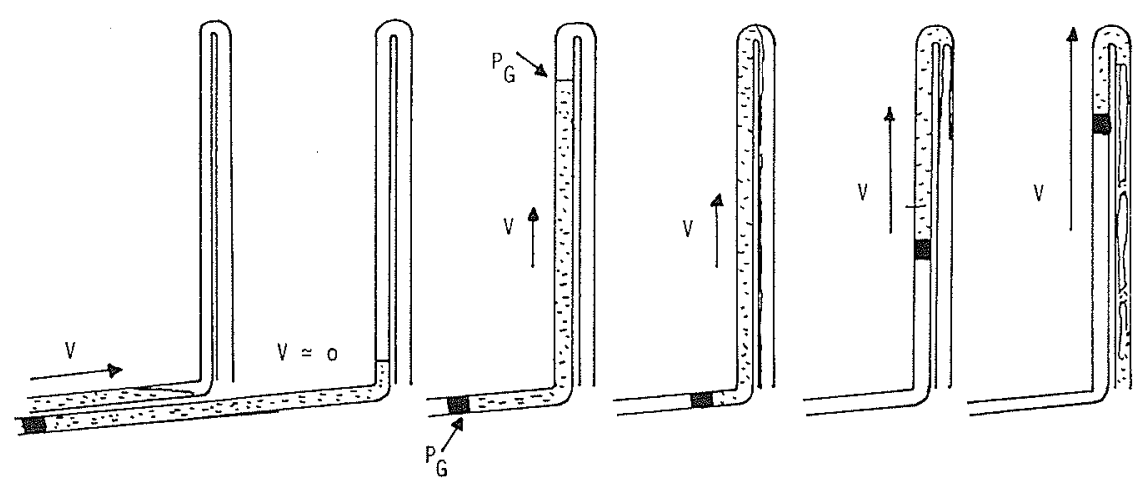

Figure 9. - Mouvement d'un bouchon đe raclage dans un U inversé (station intermédiaire).

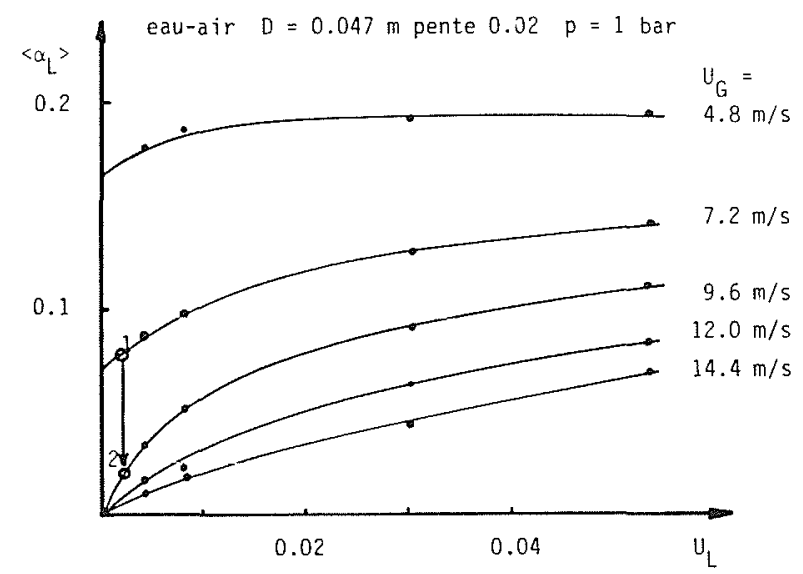

Figure 10, - Contenu liquide diphasique d'après [27]. Dans la transition $1 \rightarrow 2$ indiquée, le volume recueilli est important.

d'écoulement distincts :

1 - calcul du taux de présence de liquide, film et gouttelettes et du gradient de pression d'un tronçon à pente donnée en régime permanent ;

2 - calcul du volume du film de liquide, du débit de gouttelettes à la sortie, du débit de gaz d'une ligne pour une différence de pression donnée et un profil longitudinal imposé ;

3 -- calcul du remplissage instationnaire d'une ligne (après un râclage), compte tenu de l'entraînement de gouttelettes et éventuellement des variations de débit et de pression ;

4 - calcul du retour au régime permanent après une variation de débit ou de pression. Evaluation du volume éventuellement recueilli en bout de ligne (exemple fig. 10) ;

5 - conditions d'apparition de bouchons dans les lignes et évaluation de la fréquence et du débit instantané de liquide ;

6 - mouvement d'un bouchon de raclâge ; temps de transit ; ascension dans un riser (ce calcul nécessite celui du mouvement instationnaire du gaz dans toute la ligne).

\section{Méthodes et conclusions}

Tous les écoulements diphasiques sont traités à l'aide des théorèmes généraux décrits dans [1]. La pression diphasique [16] [17] moyenne $\overline{\bar{p}}_{G} \alpha_{G}+\bar{p}_{L} \alpha_{L}-\overline{\bar{\sigma}} \alpha_{i}$ est en général prise égale à une pression moyenne $\bar{p}$ dont on suppose qu'elle a un gradient identique aux pressions phasiques $\overline{\bar{p}}_{G}$ ou $\overline{\bar{p}}_{L}\left({ }^{2}\right)$.

Les écoulements à bouchons sont traités par l'introduction d'une loi déterministe des vitesses des poches du type (2). Il convient de noter que $U_{\infty}$ n'est pas nul même pour un écoulement horizontal [18] [4] [13].

(2) Cette hypothèse ne peut être gardée lorsqu'on traite le ressaut aéré qui se produit à l'arrière d'une poche de gaz. 
Comme on ne sait pas actuellement prédire le taux de présence de bulle $\left\langle\alpha_{B}\right\rangle$, ni le taux de présence des poches $\alpha_{0}$, on introduit des valeurs expérimentales dans le calcul. On peut alors déduire les vitesses débitantes moyennes dans le bouchon et dans le film liquide et le gradient de pression en tenant compte de l'accélération [19] [14].

Les écoulements stratifiés et annulaires sont modélisés de façon analogue. La théorie générale des écoulements stratifiés (lois dites "isomorphes" car elles sont indépendantes de la section [20]) permet de prédire le taux de présence de liquide et le gradient de pression en écoulement établi si l'on se donne une loi de rugosité uniforme équivalente de la zone interfaciale. Cette loi peut être empirique [21] [22] ou expérimentale [12] [23], ou déterminée par ajustage. On fait en général l'hypothèse d'une interface plane en moyenne mais on peut tenir compte de la convexité de l'interface. De plus, l'application aux écoulements annulaires ne présente pas de difficulté. En introduisant un coefficient de débit relatif de gouttelettes [11] on peut résoudre le problème complètement dans les cas : écoulement laminaire du gaz et du liquide [24], turbulent gaz-laminaire liquide (interface plane ou circulaire), turbulent gaz-turbulent liquide, avec ou sans entrainement [1] [20] [9] [27]. On peut alors résoudre à l'aide de ces résultats les cas 1 et 2 ci-dessus, on peut traiter aussi les cas 3 et 4 si l'on connait la loi du taux d'entrainement de gouttelettes que l'on peut déduire du coefficient de débit ou de valeurs expérimentales [25]. Le problème 5 n'est pas bien résolu à l'heure actuelle malgré certaines tentatives [26]. Le problème 6 est traité par couplage du mouvement instationnaire isotherme du gaz dans les lignes amont et aval du bouchon de râclage et du mouvement du bouchon ; celui-ci a une masse variable, lentement lors de l'ascension d'une pente, rapidement lors du déversement du liquide en un point haut. Il ne s'agit pas à proprement parler d'un problème diphasique excepté pour le déversement où il $y$ a risque d'engorgement (apparition de bouchons) de la ligne descendante [9].

Dans l'ensemble, on peut considérer que les écoulements à bouchons à fort nombre de Bond sont mal connus du fait de l'influence de la physique des interfaces sur l'aération du liquide. Les écoulements stratifiés ou annulaires sont a priori plus simples à prévoir. Il reste néanmoins à préciser les lois du frottement interfacial, les variations périmétriques des contraintes pariétales, les lois de l'entrainement de gouttelettes et les phénomènes d'instabilité conduisant à l'engorgement.

\section{Nomenclature}

$A$

$B$

$D$

$g \quad$ accélération de la pesanteur

$L \quad$ longueur de conduite

$\widetilde{L}_{P}, \widetilde{L_{B}}$ longueur moyenne des poches, des bouchons

$p, L_{B}$ pression moyenne
$\overline{\bar{p}}_{G}, \overline{\bar{p}}_{L}$ pression phasiques moyennes, gaz, liquide $U$ vitesse des poches

$U_{G}, U_{L}$ vitesses débitantes du gaz, du liquide

$U_{B} \quad$ vitesse débitante du liquide dans un bouchon

$V_{\infty} \quad$ vitesse relative des bulles par rapport au liquide (moyenne)

$V_{L} \quad$ volume de liquide d'une ligne

$\alpha_{G}, \alpha_{L}$ taux de présence local moyen du gaz, du liquide

$\alpha_{B} \quad$ taux de présence local moyen des bulles relatif au liquide

$\alpha_{P} \quad$ taux de présence local moyen des poches de gaz

$\alpha_{0} \quad$ maximum de $\alpha_{P}$

$\alpha_{i} \quad$ taux de présence d'interface [1] [16]

$\mu_{G}, \mu_{L}$ viscosités dynamiques du gaz, du liquide

$\nu_{G}, \nu_{L}$ viscosités cinématiques du gaz, du liquide

$\rho_{G}, \rho_{L}$ masses volumiques du gaz, du liquide

$\stackrel{c}{\sigma}$ tension interfaciale

$\overline{\bar{\sigma}} \quad$ tension interfaciale moyenne

$\tau_{0} \quad$ contrainte pariétale

$<>$ moyenne sur une section

\section{Bibliographie}

[1] FITREMANN J.M. - Thèse de Doctorat, Paris, 1977,

[2] ROS J.C. - J. Petr. Techn. p. 1037, Oct. 1961

[3] TRIKI T. - Thèse de Docteur-ingénieur, Paris, 1978.

[4] ZUKOSKI E. - J. Fluid Mech, p. 821, 25, 4, 1966.

[5] GALAUP J.P. - Thèse de Docteur-Ingénieur, Grenoble 1975.

[6] RESCH F. - SHF Session $N^{\circ} 112, a-4,1979$.

[7] POSTAIRE J.G. et FITREMANN J.M. - IEEE, IM 26 , $\mathrm{N}^{\circ} 3,1977$.

[8] FITREMANN J.M., COGNET G., TRIKI T., SOUHAR M., KOECK C. - ATP-CNRS 2537, DIP 103/78, 1978.

[9] FITREMANN J.M., GENIN R. - rapport DIP 101/79.

[11] WALLIS G.B. - One dimensional Two-Phase Flow, Mc Graw Hill, 1969

[12] MOECK E., STACHIEWICZ J. - Int. J. Heat Mass Transfer, $15,1962$.

[13] FERRE D. - Thèse de $3^{\text {ème }}$ cycle, Paris, 1978.

[14] FERRE D. - article Revue de l'IFP, 1979.

[15] FERRE D. - SHF Session N ${ }^{9} 112, \mathrm{~B}-3,1979$.

[16] FITREMANN J.M. - The basic theory of twophase hydrodynalics, soumis au Journal de Mécanique.

[17] LHUILLIER D. - Hydrodynamic description of laminar two-phase flow with phase changes, soumis à $I$. J. Multiphase Flow.

[18] FITREMANN J.M. - $2^{\mathrm{e}}$ Congrés Français de Mécanique, Toulouse, 1975.

[19] FITREMANN J.M. - Revue de l'TFP, XXX, N 2, 1975.

[20] FITREMANN J.M., FERRE D., GUILPIN C., CROUZIER O. - Rapport DIP 204/78.

[21] CHARNOCK H. - Quart. J.R. Meteorological Soc., p. 639, $81,1955$.

[22] LARSON T., WRIGHT J.W. - J. Fluid Mech. p. 417, 70, 3, 1975.

[23] SUZANNE C. - Thèse de Docteur-Ingénieur, Toulouse, 1977.

[24] YU H.S., SPARROW E.M. - Stratified Laminar Flow in Ducts of Arbitrary Shape, AICHE Journal, p. 10, January 1967.

[25] COUSINS L.B., HEWITT G.F., AERE R. - 56-57, Harwell, 1962.

[26] DUKLER A., TAITEL Y. - AICHE J., p. 47, 22, 1, 1976.

[27] CROUZIER O. - Thèse de $3^{\mathrm{e}}$ cycle, Paris, 1978. 


\title{
Discussion
}

\author{
Président : M. le Professeur FORTIER
}

M. le Président. - Je remercie M. FITREMANN. Y-a-t-il des questions à lui poser?

M. MONDIN. - On pourrait évoquer l'affaire d'EKOFISK. En général la méconnaissance des phénomènes diphasiques se traduit par des résultats désagréables; et là, pour une fois, on a constaté des résultats très agréables.

M. FITREMANN. - Dans le cas de la rupture de vanne à EKOFISK on pouvait s'attendre à des débits de liquide extrêmement importants. En réalité, le fait qu'on ait eu affaire à un mélange diphasique diminue beaucoup la vitesse limite que l'on obtient à partir d'un réservoir pressurisé ; dans ce cas le débit a été environ 20 fois inférieur au débit qu'on aurait pu attendre pour un écoulement monophasique et la même pression. Le résultat a donc été très agréable.

Dans les problèmes de sûreté nucléaire, en cas de brèche du circuit pressurisé d'un réacteur, cela limite aussi le débit du réfrigérant.

M. DELHAYE. - Quelle est la hauteur des puits? Quelle est la différence de pression entre le fond du puits et la sortie ?

M. FITREMANN. - Elle peut être assez grande ; on exploite couramment à $3000 \mathrm{~m}$, et cela peut aller jusqu'à $6000 \mathrm{~m}$; mais il y a aussi des gisements qui sont exploités à $100 \mathrm{~m}$ sous terre. On a parfois aussi affaire à des gisements qui sont à plusieurs profondeurs qu'on exploite par le même puits.

En valeur approchée, on a en fond de puits à peu près la pression hydrostatique équivalente d'une colonne d'eau. Pour un puits à $3000 \mathrm{~m}$ on pourrait avoir une pression dans le gisement de l'ordre de 300 bar baissant au cours de la vie du gisement. En tête de puits on a 40 bar, parfois moins.

M. DELHAYE. - Quelle est l'importance de la perte de pression par accélération?

M. FITREMANN. - Dans les puits qui sont des puits à écoulement relativement lent, c'est-à-dire en début d'exploitation du gisement, l'accélération n'est pas très forte parce que la pression est élevée et que l'écoulement n'est pas très rapide. L'écoulement est surtout à forte teneur en huile. Par contre, vers la fin de vie d'un gisement la teneur en gaz augmente (ou le puits peut être en gas-lift) et la pression baisse ; il peut en résulter une accélération importante. Cela peut intervenir dans le terme de gradient de pression pour une correction de l'ordre de 20 ou $30 \%$.

M. DELHAYE. - Le diagramme $U^{+}=f\left(U_{G}^{+}+U_{L}^{+}\right)$est analogue au diagramme de Zuber. A-t-on comparé les résultats expérimentaux directement au modèle de Zuber et Findlay (compte tenu des améliorations apportées par Ishii pour les valeurs de $C_{0}$ et de $v_{g j}$ dans les différents ty pes d'écoulement).

M. FITREMANN. - On peut se servir de modèles relativement classiques, mais disons qu'ils ne s'adaptent pas très bien aux écoulements dans ces puits, parce qu'on a un diamètre assez fort pour être en régime à poches et bulles de gaz dans les bouchons. Dans ce cas, les termes de glissement sont assez différents. Le glissement de la poche de gaz par rapport à la poche de liquide est pratiquement deux fois plus élevé que la valeur habituelle (Nicklin) : il faut donc corriger ce type de modèles pour tenir compte de ce phénomène.

M. DELHAYE. - Vous avez présenté le diagramme classique de Findlay avec la même valeur. C'est $1,2 \ldots$ pour les écoulements à forte pente.

M. FITREMANN. - Ceci concerne la vitesse des poches de gaz. Il faut tenir compte séparément du glissement des bulles. La pente de la courbe $\left(U, U_{G}+U_{L}\right)$ est voisine de celle donnée par Zuber et Findlay mais le glissement est 1,7 fois la valeur courante utilisée par ces auteurs.

M. DODU. - J'ai été frappé par la description donnée par M. FITREMANN des régimes d'écoulements et notamment par la présence des petites bulles à la partie supérieure des bouchons au-dessus des poches.

La recherche des corrélations dans ce gente de problème est souvent délicate, car il n'y a pas actuellement de certitude pour que la gravité et la tension superficielle soient les seuls paramètres externes dominants.

C'est la raison pour laquelle il me paraît opportun de mentionner le principe d'une expérimentation que nous avons entreprise à Grenoble, dans le cadre d'une étude de l'entrấnement d'air menée sous la responsabilité de M. J.M. MICHEL et au cours de laquelle nous observons des phénomènes de même genre que ceux que vous décrivez : un écoulement en canal rectangulaire à fond horizontal franchit une marche formant dénivellation du fond et détermine une cavité dans laquelle de l'air est injecté. Il est intéressant de mentionnner que, dans cette expérience, la gravité a perdu son caractère dominant. Nous avons donc une possibilité d'expérimentation accrue sur l'entraînement d'air et la formation de bulles. En outre l'accès de la surface libre inférieure permet facilement la collecte de données expérimentales par introduction de sondes à travers le plancher du canal. Dans les investigations expérimentales que nous tenterons, nous nous proposons de rechercher si la microgéométrie et la microcinématique de l'écoulement ont aussi une influence sur. l'entraînement d'air et les caractéristiques globales de l'écoulement diphasique.

Dans ce sens, il me semble que nos travaux et ceux de M. FITREMANN relèvent de préoccupations communes.

M. FITREMANN. - Je crois effectivement que ces préoccupations d'entrainement de gaz sont assez répandues.

Je peux dire deux choses:

D'abord, l'effet de gravité dont vous parlez n'influence pas beaucoup sur le phénomène. M. FERRE vous montrera cet après-midi un écoulement dans lequel il se produit la même chose. Par conséquent on rencontre aussi des cas où le phénomène n'est pas influencé par l'accélération de la pesanteur.

D'autre part M. RESCH a été l'un des premiers à analyser ce mécanisme d'entrânement dans un ressaut hydraulique.

Ici, dans tous ces écoulements on a un phénomène très similaire à celui du ressaut hydraulique, avec les mêmes difficultés de modélisation et d'expérimentation.

M. DELHAYE. - Il me semble que le diamètre du tube ne doit pas intervenir directement dans les corrélations d'entrấnement de bulles derrière les bouchons. Le phénomène est directement lié à la vitesse du film.

M. FITREMANN. - Le diamètre intervient par effet de bilan. La vitesse ascensionnelle des bulles est de l'ordre de $\left(g \sigma / \rho_{L}\right)^{\mathrm{i} / 4}$

La vitesse ascensionnelle des poches de gaz est de l'ordre de $\sqrt{g} D$, par suite le débit de film dépend du diamètre et du taux de bulles dans le bouchon.

M. SEMERIA. - J'ai une remarque un peu générale à faire, et une question pour l'assistance.

Je n'ai pas assez insisté tout à l'heure peut-être, mais il m'a semblé que les phénomènes d'entraînement de gouttes à partir de films liquides et ceux du dépôt des gouttes sur les parois étaient, pour les appareils industriels, encore traités très empiriquement. Des mécanismes d'entraînement de gouttes ont été recensés, notamment dans les turbines à vapeur humide, mais nous manquons de travaux de synthèse permettant, par exemple, de mieux dimensionner les organes de séparation des émulsions liquide-gaz.

M. FITREMANN. - Ces mécanismes d'entraînement sont très mal connus.

Dans le cas de l'exploitation du pétrole il s'agit bien entendu de séparateurs qu'on dimensionne pratiquement à 
l'aide d'un seul paramètre : un temps de rétention. Cette estimation est extrêmement approximative, il faut l'avouer : et il y aurait beaucoup à faire sur ce problème pour obtenir un meilleur dimensionnement.

M. BAILLEUX. - Dans le cadre du projet d'électrolyse de l'eau, pour E.D.F. et GAZ DE FRANCE, c'est un des points du programme. On est en train de faire le tour des équipes qui pourraient donner des précisions.

M. JAFFRIN. - Vous avez montré sur un graphique que la longueur des poches était beaucoup plus grande que celle des bouchons.

Est-ce un cas fréquent, ou est-ce que cela dépend du rapport "débit de gaz/débit de liquide" ? A-t-on dans un puits de pétrole des poches beaucoup plus longues que les bouchons?

M. FITREMANN. - Le rapport peut prendre presque n'importe quelle valeur.

Au début de la formation des poches, elles se forment à partir d'un écoulement à bulles, et la longueur des poches atteint de l'ordre du diamètre, alors que la longueur du bouchon correspond à tout le reste de l'écoulement. Par contre, pour un fort taux de gaz, la longueur des poches peut atteindre 100 ou 150 diamètres, et la longueur des bouchons peut être aussi petite que 0,3 diamètre, leur durée de vie étant assez courte mais assez notable pour qu'on puisse en mesurer la vitesse sur une dizaine de diamètres de conduite.

\section{Abstract \\ Two-Phase flow prediction problems in underground hydrocarbon recovery projects}

Economical reasons favour two-phase transport in long lines, especially from marine gas/oil fields. The prediction of gas or liquid rate mean values as well as transient properties is required. Moreover, the liquid hold-up knowledge of some importance as its value (or variation in a transient) is needed for the design of separators, safety devices such as slug catchers and in a number of cases the flow configuration is also needed. Very few prediction models are accurate enough when used with field values of the physical properties.

The main problems in the case of the extraction process (oil wells) are :

- accurate prediction of head loss in vertical or inclined line (up to $45^{\circ}$ ) especially in the slug-flow regime. As the internal diameter is large, and the interfacial constant small, the Bond number

$$
\frac{\rho_{L} g D^{2}}{\sigma}
$$

is large and the prevailing configuration is that of large gas-pockets and bubble swarms in the liquid slugs. The usual formulas found in most text books for the liquid holdup in the slug flow regime are inaccurate as they do not take into account the presence of the bubbles.
- the calculation of the physical properties, usually obtained from thermodynamics analysis does not take into account the kinetics of phase-changes and can lead to errors in the flow estimates. Also, heat transfer has to be taken into account since the flow is neither isothermal nor adiabatic.

In two-phase transport lines, the main difficulty is to predict the liquid holdup in the gas-lines usually in the stratified flow regime. The very few available models have been found totally inadequate when confronted with field values. Better models are possible when taking into account interfacial friction and interface curvature, and the turbulent properties of the flow near the walls and near the interface. These methods are sufficient for steady-state and slow transient prediction.

Slug-flow occurs in oil-lines and in some occasions in gaslines. Its prediction is largely related to the gas-slug velocity correlation and the influence of the bubble swarms in the liquid slug. The friction curve behaviour has still to be explained.

For high velocity, high-pressure flow, droplet entrainment is an important phenomenon. Incipient entrainment gasvelocity is roughly known in most situations but entrainment rates are not and need further experimentation with field conditions. 